Clinical and Nutritional Care Pathways of Patients with Malignant Bowel Obstruction: A Retrospective Analysis in a Tertiary UK Center

Pinal S Patel, Konstantinos C. Fragkos Icon, Niamh Keane, Katrine Cauldwell, Francis O'Hanlon, Jennifer Rogers, Sarah Obbard, John Barragry, Gregory Sebepos-Rogers, Jane Neerkin, Shameer Mehta, Farooq Rahman \& Simon Di Caro

\begin{abstract}
We describe a retrospective cohort study of patients with malignant bowel obstruction to examine their nutritional care pathways between 1.1.16 and 31.12.16 with readmissions until 31.12.17. Data were analyzed by comparing patients who were referred $(R)$ and not referred (NR) for PN. We identified 72 patients with $117 \mathrm{MBO}$ admissions (mean \pm SD age:63.1 $\pm 13.1 \mathrm{yrs}$, 79\% female). 24/72 patients were in $R$ group. Predominant primary malignancies were gynaecological and lower-gastrointestinal cancers (76\%). 83\% patients had metastases (61\% sub-diaphragmatically). All patients were at high-risk of malnutrition and baseline mean weight loss was $7 \%$. Discussion of PN at multidisciplinary team meeting (MDT) $(22 \%$ vs.5\%, $\mathrm{P}=0.02)$ and dietetic contact ( $94 \%$ vs. $41 \%, \mathrm{P}<0.0001)$ were more likely to occur in the R group. In 13/69 MBO admissions in NR group, reasons for non-referral were unclear. Median baseline and follow-up weight was similar (55-55.8 kg). Overall survival was $4.7(1.4-15.2)$ months, with no differences by referral groups. We compared a sub-sample of patients who 'may have' required PN $(n=10)$ vs. those discharged on home PN $(n=10)$ and found greater survival in the HPN group (323vs.91 day, $\mathrm{P}<0.01$ ). Our findings highlight disparity in care pathways suggesting that nutritional care should be integrated into clinical management discussion(s) at MDT to ensure equal access to nutritional services.
\end{abstract}

\title{
Introduction
}

\section{Nutrition in Cancer}

While malnutrition and cachexia can occur at any time during the cancer journey, it is most common in the advanced stages of the disease and is multifactorial in origin (1-4). Up to 50\% of advanced cancer patients are cachectic, which if left untreated can shorten survival $(5,6)$. The seminal work by Martin and et al., showing the independent prognostic effects of BMI and weight loss in a grading system, demonstrated that the odds of mortality were two-three fold higher when BMI was low and percentage of weight loss was high (7), eg., the highest grade (weight loss of $15 \%$ and BMI $20 \mathrm{~kg} / \mathrm{m} 2$ ) was associated with the shortest survival of $4.1 \mathrm{mo}$.

The importance of nutritional therapy as an adjunct to cancer care amongst healthcare professionals remains controversial. In an Italian survey of nutritional awareness amongst 2,375 Italian oncology members, only $5.7 \%$ oncologists responded to the survey, which may reflect a lack of nutritional awareness (8). Furthermore, a survey by oncologists, found that at first oncological contact up to $64 \%$ of patients presented with 1-10kg weight loss (9) suggesting that malnutrition could be addressed earlier. Guidelines have been produced by nutrition and more recently oncology societies on nutritional support. The European Society for Parenteral and Enteral Nutrition (ESPEN) expert group recommendations for action against cancer-related malnutrition highlighted three main areas for cancer-associated malnutrition: 1) nutritional screening should be conducted earlier; 2) nutritional assessment should use all forms of measures including body composition; and 3) clinicians should use 'multi-model nutritional interventions' with a patient centered approach (1). Furthermore, a recent position paper by the European Society for Medical Oncology (ESMO), recommended guidelines for medical oncologists on nutritional care, which highlight the importance: of i) awareness of latest nutrition guidelines; ii) nutritional screening and assessment at regular intervals, and iii) that all nutritional care should be given in a multidisciplinary environment (10). The fundamental change in 
culture underpinning these guidelines is the recommendation that every cancer patient has a right to nutritional care when malnutrition is identified with regular screening and assessment.

\section{Malignant Bowel Obstruction}

Loss of nutritional autonomy due to intestinal failure (IF) often occurs as a consequence of malignant bowel obstruction (MBO). If nutritional needs are not addressed the sequalae of cancer cachexia can progress rapidly. $\mathrm{MBO}$ can occur in the small or large bowel, can be partial or total, single or multilevel, and can present intermittently $(11,12)$.

Global prevalence of $\mathrm{MBO}$ has been estimated to occur in 3-15\% (13) of all cancers with varying rates according to the underlying malignancy. The most common primary malignancies relate to gynaecological and lower GI cancers (5-51\% in ovarian cancer and $10-28 \%$ in GI cancer) and to a lesser extent extra-abdominal malignancies such as bladder cancer or breast/melanoma cancer with peritoneal metastases $(14,15)$. Often patients present several times with MBO: on average at least 1.37 times (13). Mean survival has been shown to vary considerably $(0.8-8.5 \mathrm{mo}$,) in MBO patients who have undergone palliative surgery or been managed conservatively. However, inoperable MBO without nutritional intervention can reduce survival to 4-5weeks (13). Survival of MBO patients receiving home PN (HPN) also varies significantly. A recent Cochrane review of MBO patients who were given HPN showed median survival across 13 studies of 15-155 day (range: 3 to 1278 day) (16).

Often PN is the only plausible nutritional route in $\mathrm{MBO}$, both as an inpatient and at home.

Improvements in quality of life have been observed in advanced cancer patients who have been started on HPN (17). Variation exists amongst countries worldwide, with intermediate rates reported in Spain, France, Belgium and UK (10-50\%) and high rates in Netherlands, Italy and USA ( $\geq 50 \%)(18)$. In the UK, which historically had a low incidence uptake of HPN in malignancy, there has been a significant rise in HPN registrations: 1 in 4 new HPN registrations are for patients with underlying malignancies (19). However, the use of PN still remains controversial in advanced cancer. Reasons for this may be financial (PN is expensive and considered to add to financial burden), or institutional (not all hospitals have links to an intestinal failure unit and consequently access to discharging on HPN), or due to the perceived risks of HPN (line complications, increased stay or visits to hospital), amongst others. Currently there are no formal UK guidelines for the management of $\mathrm{MBO}$ and if any nutritional care should be provided. Information on cancer patients who are screened and referred for nutritional assessment is known to some extent. However, there is an important research gap for MBO patients who are not referred for any nutritional support. We therefore aimed to examine i) what characterizes the MBO population, ii) what medical and nutritional care do patients with $\mathrm{MBO}$ who are referred or not referred for nutrition receive and iii) if any of these care pathways affect survival.

\section{Materials and Methods}

\section{Study Design and Setting}

We used a retrospective cohort design for this study. Patient data was identified at University College London Hospital (UCLH), a large teaching hospital and tertiary referral center in London, UK. All adults aged 18yrs and above admitted to UCLH with malignant bowel obstruction from 1st Jan 2016 to 31st Dec 2016 with any MBO readmissions up to 31st Dec 2017 were included.

This study is Phase I of the overall research strategy to examine nutritional care pathways in advanced cancer patients with $\mathrm{MBO}$ : in-house retrospective study of nutritional and medical care pathways at UCLH in 2016. Phase II and III are currently underway and will extend this work nationally.

\section{Patient Cohort}


To be included in the study, a patient required a cancer diagnosis and bowel obstruction during the admission identified using ICD clinical coding: C00-C99 and K56.5-K56.6, respectively. We used a definition of $\mathrm{MBO}$ as clinical or radiological evidence of MBO. Cancer during the admission includes a cancer diagnosis during anytime in the trajectory of disease with an admission to the ward (ie., not a day-case procedure or A\&E visit that did not result in an admission).

\section{Data Collection}

Data was collected using medical notes, electronic databases and in-house inpatient and HPN databases collected by the nutrition team.

\section{Basic and Medical Demographic Variables}

The following were collected: age, sex, length of admission stay, primary oncologist, primary cancer, presence and location of metastases, date of cancer diagnosis, MBO anatomy (location, single/multilevel from CT scans). Radiological evidence of MBO was primarily taken from CT scans, and if not available, an abdominal x-ray was used. Modified Glasgow prognostic (mGPS) score which reflects inflammatory status using albumin and C-reactive protein was used (20), as well as Karnofsky Performance Status (KPS) in sensitivity analyses (21).

\section{Nutrition Variables}

The following were collected: dietetic input given and date to first dietetic input; nutrition screening score on admission (UCLH nutrition screening tool-eight point scale with scores 3-6 indicating medium risk of malnutrition and $\geq 7$ as high risk of malnutrition); anthropometric indices (usual weight, height, usual BMI, weight at MBO admission, follow-up weight at 0-3 mo, and 3-6 mo,); whether a referral was made to the nutrition team for PN (reasons for any delays in referring, duration of inpatient PN, and whether HPN was set up).

\section{Biochemical Variables}

Biochemistry between 1 and 3 day of admission included: C-reactive protein, albumin, hemoglobin, white cell count, urea, creatinine, sodium, potassium, calcium, magnesium, phosphate. Levels of cancer markers during or close to the admission included: Ca-125, Ca-19-9, Ca 15-3, and cancer embryonic antigen.

\section{Care Pathways}

Information was collected about cancer treatment prior and/or during the inpatient stay, which included radiotherapy, chemotherapy, referral to surgeons and if any surgery or stent placement was performed for MBO during the admission.

Conservative management was considered non-surgical intervention, including insertion of a stent, a ryles tube or venting gastrostomy tube for decompression and symptom control, whether the patient was made nil by mouth, use of high dose steroids (defined as parenteral dexamethasone 8-16mg daily) and/or use of symptom control medications (15). These included parenteral opioids and anti-emetics, parenteral anti-secretories (hyoscine butylbromide or octreotide) and laxatives. Medications were included if they were prescribed during the admission for $\mathrm{MBO}$ and resolution of symptoms, death or discharge, though those prescribed routinely at the end of life were excluded. Data was collected to describe referral to palliative care, primary oncologist (named as oncologist 1-17), discharge location and if known, cause of death.

\section{Survival Analysis}


Survival from date of admission to death and at 3-, 6-, 12-month follow-up was examined. The censor date was 31.8.18.

\section{Statistical Analysis}

Medical and nutritional care pathway characteristics were summarized using means (SD), medians (IQR) and frequencies (percentages). Where appropriate, t-test, Kruskal-Wallis test and Chi-square respectively were used, to test for any differences between those who were referred and not referred for PN. Survival was examined with a Cox-regression using a univariate approach to identify risk factors statistically significantly associated with survival with any key risk factors then used in the multivariable Cox-regression model. Kaplan-Meier curves and log rank test for equality were used to graphically present survival of key risk factors. In secondary analyses, the reasons for referral or non-referral for PN and whether these were appropriate were further examined. In addition, we also conducted sensitivity analyses examining the non-referral PN group who we speculate 'may have' required PN (based on: i) multiple MBO admissions, ii) ward dietitian querying PN but no discussion stated in medical notes by the medical team and iii) nil nutritional intake for $>7$ days of their admission) vs. those who were discharged on HPN. All descriptive statistical analyses were conducted using STATA version 14 [Statacorp Texas USA] and survival analyses and Kaplan-Meier curves were conducted in R Statistical Software (Foundation for Statistical Computing, Vienna, Austria).

\section{Ethical Permission}

An ethical waiver was provided, by the research and development committee of University College London Hospital, as this work was considered an audit of clinical practice and registered as an audit.

\section{Results}

\section{Patient Population}

200 admissions with MBO codes were retrieved from clinical coding. Each admission's discharge summary was reviewed to verify that the patient was admitted with MBO. Of these, 128 were excluded due to the following reasons: adhesional bowel obstruction due to previous surgery, no cancer or no evidence of bowel obstruction or had an initial admission in 2015 and discharge in 2016, day-case or A\&E admission only. The nutrition team HPN database was cross-checked for any patients who may not have been identified by coding who had been discharged on HPN with bowel obstruction. This left 72 patients admitted with MBO in 2016 with 117 admissions up till the end of 2017 (Supplementary Figure 1).

\section{Baseline Characteristics of the Patient Cohort}

There were 117 admissions from 72 patients with a mean age $63.1 \mathrm{yrs}$ (SD: $13.1 \mathrm{yrs}$ ) and 79\% female. The primary cancer etiologies included gynaecological and lower-gastrointestinal malignancies, the remaining $24 \%$ had upper gastrointestinal, HPB, urological, haematological, or breast cancers. Metastases were observed in $83 \%$ of all patients, with location of metastases more likely to occur below (61\%) or above and below the diaphragm (19\%). Duration of cancer varied from 0 to 33 years (there were three patients whose primary malignancy was 16-33 years prior to admission with relapsing or metastasizing disease causing MBO in their admission in 2016) (Table 1).

Table 1. Demographics of the cohort by patient. 


\begin{tabular}{|c|c|c|c|c|}
\hline Baseline characteristics & $\begin{array}{c}\text { Total no. of patients } \\
\qquad n=72\end{array}$ & $\begin{array}{l}\text { Referred for PN } \\
\quad n=24\end{array}$ & $\begin{array}{c}\text { Not referred for PN } \\
\quad n=48\end{array}$ & P-value \\
\hline Age on admission (yrs), mean(SD) & $63.1(13.1)$ & $60.4(13.8)$ & $64.5(12.6)$ & $P=0.213$ \\
\hline Sex - Female $(n, \%)$ & $57(79.2)$ & $17(70.8)$ & $40(83.3)$ & $P=0.218$ \\
\hline Primary oncologist at UCLH $(n, \%)$ & & & & $P=0.453$ \\
\hline Oncologist 1 & $19(26)$ & $9(38)$ & $10(21)$ & \\
\hline Oncologist 2 & $8(11)$ & $2(8)$ & $6(13)$ & \\
\hline Oncologist 3 & $8(11)$ & $4(17)$ & $4(8)$ & \\
\hline Oncologist 4 & $5(7)$ & $1(4)$ & $4(8)$ & \\
\hline Oncologist 5 & $8(11)$ & $0(0)$ & $8(17)$ & \\
\hline Oncologist 6 & $4(6)$ & $2(8)$ & $2(4)$ & \\
\hline (Others < $<$ patients: Oncologist 7-17, unknown), & $20(28)$ & $6(25)$ & $14(29)$ & \\
\hline Primary malignancy $(n, \%)$ & & & & $P=0.217$ \\
\hline Gynaecology & $36(50.0)$ & $9(37.5)$ & $27(56.3)$ & \\
\hline Lower Gl & $19(26.4)$ & $8(33.3)$ & $11(22.9)$ & \\
\hline HPB & $3(4.2)$ & $0(0.0)$ & $3(6.3)$ & \\
\hline Upper GI & $3(4.2)$ & $2(8.3)$ & $1(2.1)$ & \\
\hline Urology & $2(2.8)$ & $0(0.0)$ & $2(4.2)$ & \\
\hline Haematology & $2(2.8)$ & $1(4.2)$ & $1(2.1)$ & \\
\hline Breast & $1(1.4)$ & $0(0.0)$ & $1(2.1)$ & \\
\hline Other & $6(8.3)$ & $4(16.7)$ & $2(4.2)$ & \\
\hline Time since cancer diagnosis & $\mathrm{n}=71$ & $\mathrm{n}=24$ & $\mathrm{n}=47$ & $P=0.217$ \\
\hline Years & $1.31(0-33.3)$ & $0.91(0-33.3)$ & $1.38(0.0-16.6)$ & \\
\hline Months & $15.7(0-400)$ & $11.2(0-400)$ & $16.4(0-199.2)$ & \\
\hline Patients with 2 primary malignancies ( $n, \%)$ & $6(8.3)$ & $1(4.2)$ & $5(10.4)$ & $P=0.366$ \\
\hline Presence of metastases $(n, \%)$ - Yes & $60(83.3)$ & 19 (79.2) & $41(85.4)$ & $P=0.502$ \\
\hline Location of metastases $(n, \%)$ & & & & $\mathrm{P}=0.676$ \\
\hline Lower diaphragm & $44(61.1)$ & $15(62.5)$ & $29(60.4)$ & \\
\hline Upper diaphragm & $2(2.8)$ & $0(0.0)$ & $2(4.2)$ & \\
\hline Upper and Lower diaphragm & $14(19.4)$ & $4(16.7)$ & $10(20.8)$ & \\
\hline Prior surgery for cancer $(n, \%)-$ Yes & $32(44.4)$ & $9(37.5)$ & $23(47.9)$ & $P=0.402$ \\
\hline Prior radiotherapy $(n, \%)$ - Yes & $17(23.6)$ & $7(25.0)$ & $11(22.9)$ & $P=0.844$ \\
\hline Prior chemotherapy $(n, \%)$ - Yes & $52(72.2)$ & $18(75.0)$ & $34(70.8)$ & $P=0.710$ \\
\hline
\end{tabular}

Values are presented as medians with range (minimum-maximum) in parentheses unless otherwise stated.

P-values denote differences between the two groups referred and not referred for PN, using ttest for parametric variables, Kruskal-Wallis test for nonparametric variables and Chi2 test for categorical variables.

There were no significant differences in any baseline characteristic by whether patients were referred for PN. Several Oncologists (Oncologist 1, 3 and 6) had a relatively equal no. of patients they referred and not referred for $\mathrm{PN}$, while it was more likely for patients under Oncologist 2, 4, and five to not be referred for PN. Of 69,000 admissions to UCLH with a cancer coding in 2016, the prevalence of MBO was $0.1 \%$.

\section{Medical Care Pathways}

In the majority of admissions, a CT scan was performed to diagnose MBO ( $82 \%$ of all admissions), occurring on the day or day after admission. Overall patients were more likely to present with single compared to multi-level MBO, and more likely to occur in the small than large bowel with higher proportion of small MBO in those referred for PN (Table 2).

Table 2. Medical care pathways received during each admission. 


\begin{tabular}{|c|c|c|c|c|}
\hline Medical treatment & $\begin{array}{l}\text { Total no. of admissions } \\
\qquad n=117\end{array}$ & $\begin{array}{l}\text { Referred for PN } \\
n=48\end{array}$ & $\begin{array}{l}\text { Not referred for PN } \\
\quad n=69\end{array}$ & P-value \\
\hline Palliative inpatient chemotherapy received during admission ( $n, \%)-Y e s$ & $40(34.2)$ & $23(47.9)$ & $17(24.6)$ & $P=0.009$ \\
\hline No. of inpatient chemotherapy infusions received & $\begin{array}{l}n=41 \\
1(1-6)\end{array}$ & $\begin{array}{l}n=23 \\
1(1-6)\end{array}$ & $\begin{array}{l}n=18 \\
1(1-2)\end{array}$ & $P=0.012$ \\
\hline Time to first inpatient chemotherapy infusion from admission (days) & $8(0-39)$ & $9(0-39)$ & $7(1-27)$ & $P=0.607$ \\
\hline Inpatient Radiotherapy received (n, \%) - Yes & $1(0.9)$ & $1(2.1)$ & $0(0.0)$ & $P=0.229$ \\
\hline Surgeon input received $(n, \%)$ - Yes & $59(50.4)$ & $28(58.3)$ & $31(44.9)$ & $P=0.154$ \\
\hline Time to surgeon input from admission (days) & $\begin{array}{c}n=59 \\
1(0-17)\end{array}$ & $\begin{array}{l}n=28 \\
1(0-9)\end{array}$ & $\begin{array}{c}n=31 \\
1(0-17)\end{array}$ & $P=0.632$ \\
\hline Surgery for bowel obstruction $(n, \%)$ - Yes & $15(12.8)$ & $11(22.9)$ & $4(5.8)$ & $P=0.006$ \\
\hline Time to surgery from admission (days) & $8(0-45)$ & $11(1-45)$ & $1(0-1)$ & $P=0.007$ \\
\hline Referral to Palliative care $(n, \%)$ - Yes & $\begin{array}{c}n=99 \\
48(48.5)\end{array}$ & $\begin{array}{c}n=41 \\
19(46.3)\end{array}$ & $\begin{array}{l}n=58 \\
9(50.0)\end{array}$ & $P=0.994$ \\
\hline Time to palliative care referral from admission (days) & $\begin{array}{c}n=45 \\
4(0-99)\end{array}$ & $\begin{array}{c}n=18 \\
3(0-99)\end{array}$ & $\begin{array}{c}n=27 \\
4(0-34)\end{array}$ & $P=0.568$ \\
\hline Conservative management $(n, \%)$ & $102(87.2)$ & $37(77.1)$ & $65(94.2)$ & $P=0.006$ \\
\hline Duodenal stent $(n, \%)$ - Yes & $1(0.9)$ & $1(2.1)$ & $0(0.0)$ & $P=0.229$ \\
\hline Colonic stent $(n, \%)-$ Yes & $15(12.8)$ & $2(4.2)$ & $13(18.8)$ & $P=0.020$ \\
\hline Ryles tube inserted $(n, \%)$ - Yes & $\begin{array}{c}n=111 \\
51(45.9)\end{array}$ & $\begin{array}{c}n=48 \\
27(56.3)\end{array}$ & $\begin{array}{c}n=63 \\
24(38.1)\end{array}$ & $P=0.018$ \\
\hline Venting gastrostomy discussed (n, \%) - Yes & $\begin{array}{c}n=101 \\
4(4.0)\end{array}$ & $\begin{array}{l}n=43 \\
3(7.0)\end{array}$ & $\begin{array}{l}n=58 \\
1(1.7)\end{array}$ & $P=0.409$ \\
\hline Venting gastrostomy inserted $(n, \%)$ - Yes & $1(1.0)$ & $1(2.3)$ & - & - \\
\hline Multi or Single level bowel obstruction $(n, \%)$ & & & & $P=0.454$ \\
\hline Multi & $45(38.4)$ & $19(39.6)$ & $26(37.7)$ & \\
\hline Single & $56(47.9)$ & $25(52.1)$ & $31(44.9)$ & \\
\hline Unclear & $14(12.0)$ & $3(6.2)$ & $11(15.9)$ & \\
\hline No BO $(n, \%)$ & $2(1.7)$ & $1(2.1)$ & $1(1.5)$ & \\
\hline Small or Large bowel obstruction $(n, \%)$ & & & & $P=0.011$ \\
\hline Small & $67(57.3)$ & $36(75.0)$ & $31(44.9)$ & \\
\hline Large & $21(17.9)$ & $5(10.4)$ & $16(23.2)$ & \\
\hline Small and Large & $10(8.6)$ & $4(8.3)$ & $6(8.7)$ & \\
\hline Unclear & $17(14.5)$ & $2(4.2)$ & $15(21.7)$ & \\
\hline No BO $(n, \%)$ & $2(1.7)$ & $1(2.1)$ & $1(1.5)$ & \\
\hline CT scan during or close to admission ( $n, \%)$ & & & & $P=0.207$ \\
\hline Yes & $96(82.1)$ & $43(89.6)$ & $53(76.8)$ & \\
\hline No & $9(7.7)$ & $2(4.2)$ & $7(10.1)$ & \\
\hline No-other scan & $12(10.2)$ & $3(6.2)$ & $9(13.0)$ & \\
\hline Time to $1^{\text {st }}$ CT scan (days) & $0(-32-44)$ & $1(-32-36)$ & $0(-14-44)$ & $P=0.343$ \\
\hline
\end{tabular}

Values are presented as medians with range (minimum-maximum) in parentheses unless otherwise stated.

P-values denote differences between the two groups referred and not referred for PN, using t-test for parametric variables, Kruskal-Wallis test for nonparametric variables and $\mathrm{Chi}^{2}$ test for categorical variables.

Up to $72 \%$ patients were likely to have had some form of prior cancer treatment; prior chemotherapy was more likely to be used then surgery or radiotherapy. With regards to care pathways during the inpatient stay, patients who were referred for PN were more likely to receive palliative chemotherapy then those who were not ( $P=0.009,47.9 \%$ vs. $24.6 \%$, respectively). The median number of inpatient chemotherapy infusions received were 1 (range: 1-6) in those referred and 1-2 in those not referred for PN. Similarly, those who were referred for PN were more likely to receive surgery for bowel obstruction than those not referred for PN ( $P=0.006,22.9 \%$ vs. $5.8 \%$, respectively). There was no difference in referral to palliative care by $\mathrm{PN}$ referral group. Those not referred for PN were more likely to be treated conservatively, and less likely to have colonic stent insertion than those referred for PN likely indicating that the colonic stent would resolve the MBO, hence patients not requiring PN.

Supplementary Table 1 shows the levels of routine biochemistry measurements on admission and recent cancer markers. There were no statistically significant differences in biochemistry by PN referral group (except for urea), including modified Glasgow prognostic score. Overall, amongst all admissions, most patients presented with a high CRP (median $53.4 \mathrm{mmol}$ ) and borderline low albumin (median $36 \mathrm{mmol} / \mathrm{L}$ ), resulting in approximately $85 \%$ of the patient sample in mGPS scores 2 or 3 (44.8\% mGPS score 2 and $39.6 \%$ mGPS score 3). The cancer markers were measured in smaller proportions of the cohort, and where measured varied significantly by patient (eg., Ca-125 range: 9-16, 813 kIU/L).

\section{Nutritional Care Pathways}

Table 3 presents nutritional care received during each admission. If PN was discussed at MDT (22.2\% vs. $4.8 \%, \mathrm{P}=0.020$ ) or a patient had dietetic contact, they were more likely to be in the referred vs. not referred for PN group (93.8\% vs. 40.6\%). Patients who were referred for PN were assessed by the nutrition team on average on the day of referral (range: 0-6days). 
Table 3. Nutritional care pathways received during each admission.

\begin{tabular}{|c|c|c|c|c|}
\hline Nutritional treatment & $\begin{array}{c}\text { Total no. of admissions } \\
n=117\end{array}$ & $\begin{array}{c}\text { Referred for PN } \\
\quad n=48\end{array}$ & $\begin{array}{c}\text { Not referred for PN } \\
n=69\end{array}$ & P-value \\
\hline PN discussed at MDT (n, \%) - Yes & $\begin{array}{c}\mathrm{n}=108 \\
13(12.0)\end{array}$ & $\begin{array}{c}n=45 \\
10(22.2)\end{array}$ & $\begin{array}{l}n=63 \\
3(4.8)\end{array}$ & $P=0.020$ \\
\hline Time to MDT from admission (days) & $\begin{array}{c}n=13 \\
4(-1-18)\end{array}$ & $\begin{array}{c}n=10 \\
3(-1-18)\end{array}$ & $\begin{array}{c}n=3 \\
4(1-16)\end{array}$ & $P=0.865$ \\
\hline Response time referral to assessment by nutrition team (days) & $\begin{array}{l}n=49 \\
0(0-6)\end{array}$ & $\begin{array}{l}n=48 \\
0(0-6)\end{array}$ & $\begin{array}{l}n=0 \\
0(0-0)\end{array}$ & - \\
\hline Time from admission to nutrition team referral (days) & $\begin{array}{c}n=48 \\
4(0-37)\end{array}$ & $\begin{array}{c}n=48 \\
4(0-37)\end{array}$ & $\begin{array}{c}n=0 \\
0(0-0)\end{array}$ & - \\
\hline Reasons for no PN (n, \%) & - & - & & - \\
\hline$E+D$ & & & $14(20.3)$ & \\
\hline Not stated & & & $13(18.8)$ & \\
\hline No medical notes & & & $11(15.9)$ & \\
\hline BO resolved & & & $8(11.6)$ & \\
\hline EOL & & & $7(10.1)$ & \\
\hline Stented & & & $4(5.8)$ & \\
\hline Conservative management & & & $3(4.4)$ & \\
\hline Fast track discharge & & & $3(4.4)$ & \\
\hline Clinical deterioration & & & $2(2.9)$ & \\
\hline Discharged after 1 day & & & $1(1.5)$ & \\
\hline Has enteral access & & & $1(1.5)$ & \\
\hline Intermittent POI & & & $1(1.5)$ & \\
\hline Medical team did not think PN appropriate & & & $1(1.5)$ & \\
\hline \multicolumn{5}{|l|}{ Reasons for any delays in PN $(n, \%)$} \\
\hline No delays & & $23(47.9)$ & & \\
\hline No medical notes & & $3(6.3)$ & & \\
\hline Reasons not stated/not thought about & & $4(8.3)$ & & \\
\hline Not managing enough orally (partial BO) & & $1(2.1)$ & & \\
\hline Advised to trial ONS/ENS first & & $2(4.2)$ & & \\
\hline Cannot reach team & & $1(2.1)$ & & \\
\hline Initially treating BO conservatively & & $5(10.4)$ & & \\
\hline Line issue & & $6(12.5)$ & & \\
\hline Referred after $\mathrm{CT}$ results & & $1(2.1)$ & & \\
\hline Sepsis & & $1(2.1)$ & & \\
\hline Patient declined & & $1(2.1)$ & & \\
\hline PN received & & $\mathrm{n}=44$ & & \\
\hline Duration of inpatient PN (days) & - & $\begin{array}{c}14.5 \\
(0-114)\end{array}$ & - & - \\
\hline \multicolumn{5}{|l|}{ Reason for stopping inpatient PN (n, \%) } \\
\hline$E+D$ & & $12(25.0)$ & & \\
\hline HPN & & $20(41.7)$ & & \\
\hline EOL & & $6(12.5)$ & & \\
\hline Transfer to another hospital & & $2(4.2)$ & & \\
\hline Line infection & & $2(4.2)$ & & \\
\hline Pt wishes to go to home country & & $1(2.1)$ & & \\
\hline Acute deterioration & & $1(2.1)$ & & \\
\hline N/A (patients referred but not started on PN) & & $4(8.3)$ & & \\
\hline Location of discharge $(n, \%)$ & & & & $P=0.423$ \\
\hline Home & $81(69.2)$ & $33(68.8)$ & $48(69.6)$ & \\
\hline Hospice & $17(14.5)$ & $6(12.5)$ & $11(15.9)$ & \\
\hline Nursing/care home & $2(1.8)$ & $1(2.1)$ & $1(1.5)$ & \\
\hline Other Hospital & $4(3.4)$ & $3(6.3)$ & $1(1.5)$ & \\
\hline Other Country & $1(0.9)$ & $1(2.1)$ & $0(0.0)$ & \\
\hline RIP & $12(10.3)$ & $4(8.3)$ & $8(11.6)$ & \\
\hline
\end{tabular}

Values are presented as medians with range (minimum-maximum) in parentheses unless otherwise stated.

P-values denote differences between the two groups referred and not referred for PN, using Kruskal-Wallis test for non-parametric variables and Chi2 test for categorical variables.

Four patients who were referred for PN did not start PN for the following reasons: patient declined, nutrition team advised on a trial oral/enteral nutrition first, nutrition team advised management of high output stoma, and patient was stented. For the non-referred PN group, the main reasons for not being referred for PN included: patients were eating and drinking (20.3\%), BO resolved (11.6\%), or patients were end of life (10.1\%). In $18.8 \%$ no reason was stated in the medical notes, and medical notes were not available in $15.9 \%$ of admissions.

For the 44 admissions where patients received PN the median duration of PN in hospital was 14.5 day (range: 0-114 day). Delays in referrals for PN included: initially treating BO conservatively (10.4\%), central venous catheter issues (12.5\%) which mainly related to organizing access for PN, no reasons stated for delays (8.3\%). The main reasons for stopping inpatient PN included patients started eating and drinking (ie., BO resolved) (25.0\%), patients went home on PN (41.7\%), or patients were end of life (12.5\%). Patients who were referred for PN were most likely to be discharged home or to a hospice. 
Anthropometric measures and screening tool scores conducted at each admission are presented in Table 4. All patients presented at high risk of malnutrition with a median nutritional screening score of 8. Median time for nutritional screening tool to be conducted was within $24 \mathrm{hrs}$ of admission. There were no significant differences in anthropometric measures by PN referral groups and most patients had a normal BMI $(21 \mathrm{~kg} / \mathrm{m} 2$, normal range in adults: $18.5-24.9 \mathrm{~kg} / \mathrm{m} 2)$. However, the median percentage weight loss in the preceding 3-6months was 7\%. In addition, weight at 0-3month and 4-6month followup were similar by PN referral groups.

Table 4. Dietetic input and anthropometric measures during each admission.

\begin{tabular}{|c|c|c|c|c|}
\hline Nutritional treatment & $\begin{array}{c}\text { Total no. of admissions } \\
n=117\end{array}$ & $\begin{array}{l}\text { Referred for PN } \\
\quad n=48\end{array}$ & $\begin{array}{l}\text { Not referred for PN } \\
\qquad n=69\end{array}$ & P-value \\
\hline Dietetic input received $(n, \%)$ - Yes & $73(62.4)$ & $45(93.8)$ & $28(40.6)$ & $P=0.000$ \\
\hline Time to dietetic input from admission (days) & $5(0-43)$ & $4(0-40)$ & $7(0-43)$ & $P=0.008$ \\
\hline Ward dietetic input received $(n, \%)$ - Yes & $32(43.8)$ & $4(100.0)$ & $28(40.6)$ & $P=0.020$ \\
\hline NST score $(n, \%)$ & $\begin{array}{c}n=88 \\
8(0-16)\end{array}$ & $\mathrm{n}=39$ & $\begin{array}{c}n=49 \\
8(0-16)\end{array}$ & $P=0.288$ \\
\hline Time to NST score from admission (days) & $\begin{array}{c}n=88 \\
0(0-17)\end{array}$ & $\begin{array}{c}n=39 \\
1(0-17)\end{array}$ & $\begin{array}{l}\mathrm{n}=49 \\
0(0-5)\end{array}$ & $P=0.503$ \\
\hline Usual Weight (kg) & $\begin{array}{c}\mathrm{n}=95 \\
59 \\
(41.8-108)\end{array}$ & $\begin{array}{c}\mathrm{n}=46 \\
58.9 \\
(45-93.2)\end{array}$ & $\begin{array}{c}\mathrm{n}=49 \\
59 \\
(41.8-108)\end{array}$ & $P=0.549$ \\
\hline Usual BMI $\left(\mathrm{kg} / \mathrm{m}^{2}\right)$ & $\begin{array}{c}\mathrm{n}=96 \\
23.0 \\
(15.5-40.6)\end{array}$ & $\begin{array}{c}\mathrm{n}=47 \\
22.8 \\
(15.5-33.7)\end{array}$ & $\begin{array}{c}\mathrm{n}=49 \\
23.0 \\
(15.9-40.6)\end{array}$ & $P=0.642$ \\
\hline Weight at bowel obstructive episode (kg) & $\begin{array}{c}\mathrm{n}=105 \\
54.9 \\
(38-100)\end{array}$ & $\begin{array}{c}\mathrm{n}=47 \\
53.9 \\
(41.8-89)\end{array}$ & $\begin{array}{c}\mathrm{n}=58 \\
57 \\
(38-100)\end{array}$ & $P=0.145$ \\
\hline BMI at bowel obstructive episode $\left(\mathrm{kg} / \mathrm{m}^{2}\right)$ & $\begin{array}{c}\mathrm{n}=104 \\
21.1 \\
(14.8-34.9)\end{array}$ & $\begin{array}{c}\mathrm{n}=47 \\
21.0 \\
(15.0-32.6)\end{array}$ & $\begin{array}{c}\mathrm{n}=57 \\
21.2 \\
(14.8-34.9)\end{array}$ & $P=0.295$ \\
\hline Percentage weight loss from usual weight (\%) & $\begin{array}{c}\mathrm{n}=94 \\
-7.1 \\
(-45.5-26.2)\end{array}$ & $\begin{array}{c}\mathrm{n}=46 \\
-7.0 \\
(-25.8-14.3)\end{array}$ & $\begin{array}{c}\mathrm{n}=48 \\
-7.1 \\
(-45.5-26.2)\end{array}$ & $P=0.907$ \\
\hline Weight at $0-3$ month follow-up (kg) & $\begin{array}{c}\mathrm{n}=60 \\
54.6 \\
(39.9-84.1)\end{array}$ & $\begin{array}{c}\mathrm{n}=31 \\
54.0 \\
(39.9-82.8)\end{array}$ & $\begin{array}{c}\mathrm{n}=29 \\
55.0 \\
(44.6-84.1)\end{array}$ & $P=0.122$ \\
\hline Weight at 4-6month follow-up (kg) & $\begin{array}{c}\mathrm{n}=39 \\
55.8 \\
(38.7-87.3)\end{array}$ & $\begin{array}{c}\mathrm{n}=22 \\
55.7 \\
(38.7-85.4)\end{array}$ & $\begin{array}{c}\mathrm{n}=17 \\
55.8 \\
(44.5-87.3)\end{array}$ & $P=0.766$ \\
\hline
\end{tabular}

Values are presented as medians with range (minimum-maximum) in parentheses unless otherwise stated.

P-values denote differences between the two groups referred and not referred for PN, using Kruskal-Wallis test for non-parametric variables and Chi2 test for categorical variables.

Sensitivity Analyses

In sensitivity analyses (Table 5), we examined the non-referral PN group further to ascertain if there were any reasons patients should have been referred for PN. Of the 69 admissions where there was no referral for PN, we selected 46 admissions where no PN was received throughout the entire study period, we then selected the following patients: 16 admissions from 10 patients in which it was speculated patients should have been referred for PN. We examined the characteristics patients whom we speculate 'may have' required PN vs. those who were discharged on HPN (10 vs. 10 patients, respectively), presented in Table 5. Overall, most characteristics did not differ between the required PN and HPN groups except for prior radiotherapy which was more likely to occur in the HPN group. There were some non-statistically significant trends such as those in the HPN group were more likely to be younger, have surgery for their cancer, and a higher weight at 4-6month follow-up.

Table 5. Characteristics of patients for which PN may have been appropriate but were not referred for $\mathrm{PN}$ vs. patients who discharged on home PN. 


\begin{tabular}{|c|c|c|c|}
\hline & $\begin{array}{l}\text { Patients who may have required PN } \\
\qquad n=10\end{array}$ & $\begin{array}{l}\text { Patients discharged on HPN } \\
\qquad n=10\end{array}$ & P-value \\
\hline Age (yrs), mean (SD) & $63.2(14.0)$ & $55.3(13.9)$ & $P=0.223$ \\
\hline Sex - Female (n, \%) & $9(90.0)$ & $8(80.0)$ & $\mathrm{P}=0.531$ \\
\hline \multicolumn{3}{|l|}{ Primary oncologist at $\mathrm{UCLH},(\mathrm{n}, \%)$} & $P=0.437$ \\
\hline Oncologist 1 & $2(20.0)$ & $4(40.0)$ & \\
\hline Oncologist 2 & $1(10.0)$ & $0(0.0)$ & \\
\hline Oncologist 3 & $1(10.0)$ & $2(20.0)$ & \\
\hline Oncologist 4 & $3(30.0)$ & $1(10.0)$ & \\
\hline Oncologist 5 & $0(0.0)$ & $1(10.0)$ & \\
\hline (Others $<4$ patients: Oncologist $6-17$, unknown), & $3(30.0)$ & $2(20.0)$ & \\
\hline \multicolumn{3}{|l|}{ Primary malignancy $(n, \%)$} & $P=0.451$ \\
\hline Gynaecological & $6(60.0)$ & $5(50.0)$ & \\
\hline Gastrointestinal & $3(30.0)$ & $5(50.0)$ & \\
\hline Other & $1(10.0)$ & $0(0.0)$ & \\
\hline \multicolumn{3}{|l|}{ Stage of Cancer (n, \%) } & $P=0.141$ \\
\hline 1 & $0(0.0)$ & $0(0.0)$ & \\
\hline 2 & $0(0.0)$ & $3(30.0)$ & \\
\hline 3 & $3(37.5)$ & $1(10.0)$ & \\
\hline 4 & $5(62.5)$ & $6(60.0)$ & \\
\hline Presence of metastases $(n, \%)$-Yes & $9(90.0)$ & $10(100)$ & $P=0.305$ \\
\hline \multicolumn{3}{|l|}{ Location of metastases (n, \%) } & $P=0.506$ \\
\hline Upper diaphragm & $1(10.0)$ & $0(0.0)$ & \\
\hline Lower diaphragm & $5(50.0)$ & $7(70.0)$ & \\
\hline Upper and Lower diaphragm & $3(30.0)$ & $3(30.0)$ & \\
\hline Inpatient chemo received ( $\mathrm{n}, \%)$-Yes & $5(50.0)$ & $5(50.0)$ & $P=1.000$ \\
\hline Surgery for cancer (pre or during admission), (n, \%) -Yes & $3(30.0)$ & $7(70.0)$ & $P=0.074$ \\
\hline Surgery for BO during admission $(n, \%)$-Yes & $0(0.0)$ & $2(20.0)$ & $P=0.136$ \\
\hline Prior Radiotherapy (n, \%) -Yes & $0(0.0)$ & $4(40.0)$ & $P=0.025$ \\
\hline Conservative management $(n, \%)-Y e s$ & $10(100.0)$ & $8(80.0)$ & $P=0.136$ \\
\hline NST score (median, range) & $9(3-12)$ & $7(0-14)-n=8$ & $P=0.346$ \\
\hline \multirow[t]{2}{*}{ Usual weight $(\mathrm{kg})$} & $\mathrm{n}=8$ & & $P=0.722$ \\
\hline & $58(44-86)$ & $58(51-93)$ & \\
\hline \multirow[t]{2}{*}{ Usual BMI $\left(\mathrm{kg} / \mathrm{m}^{2}\right)$} & $n=8$ & & $P=0.722$ \\
\hline & $22.7(17.6-36.7)$ & $22.1(17.0-33.7)$ & \\
\hline \multirow[t]{2}{*}{ Weight at BO episode $(\mathrm{kg})$} & & $n=9$ & $P=0.807$ \\
\hline & $52(38-100)$ & $52(43-87)$ & \\
\hline BMI at BO episode $\left(\mathrm{kg} / \mathrm{m}^{2}\right)$ & $21.2(14.8-32.7)$ & $19.5(15.9-32.6)-n=9$ & $P=0.568$ \\
\hline Weight at $0-3$ month follow-up (kg) & $\begin{array}{c}n=6 \\
55.8(44.6-79.3)\end{array}$ & $\begin{array}{c}n=8 \\
53.2(41.8-82.8)\end{array}$ & $\mathrm{P}=0.519$ \\
\hline \multirow[t]{2}{*}{ Weight at 4-6month follow-up (kg) } & $\mathrm{n}=2$ & $n=8$ & $P=0.296$ \\
\hline & $51.6(47.4-55.8)$ & $55.7(47.5-76.9)$ & \\
\hline \multirow[t]{2}{*}{$\%$ weight loss at BO episode } & $\mathrm{n}=8$ & $n=9$ & $P=0.773$ \\
\hline & $-10.2(-33.3--0.5)$ & $-8.4(-21.0--3.3)$ & \\
\hline \multirow[t]{2}{*}{$\%$ weight loss at 3 month follow-up } & $n=6$ & $n=8$ & $P=0.070$ \\
\hline & $2(-0.3-8)$ & $-1.3(-5.9-9)$ & \\
\hline \multirow[t]{2}{*}{$\%$ weight loss at 6 month follow-up } & $\mathrm{n}=2$ & $\mathrm{n}=7$ & $P=0.770$ \\
\hline & $2.2(-1.2-5.6)$ & $4.5(-6.6-13.6)$ & \\
\hline Dietetic input - (n, \%)-Yes & $6(60.0)$ & $7(70.0)$ & $P=0.639$ \\
\hline PN discussed at MDT - (n, \%)-Yes & $0(0.0)$ & $1(10.0)$ & $P=0.305$ \\
\hline \multicolumn{3}{|l|}{ mGPS $(n, \%)$} & $P=0.244$ \\
\hline 1 & $1(12.5)$ & $7(87.5)$ & \\
\hline 2 & $4(50.0)$ & $1(12.5)$ & \\
\hline 3 & $3(37.5)$ & $0(0.0)$ & \\
\hline \multirow[t]{2}{*}{ KPS (n, \%) } & $n=6$ & $\mathrm{n}=10$ & $P=0.355$ \\
\hline & $65(60-70)$ & $60(50-70)$ & \\
\hline Length of stay (days) & $10(5-64)$ & $20(6-66)$ & $P=0.597$ \\
\hline Median no. of admissions & $1.5(1-3)$ & $2(1-6)$ & $P=0.082$ \\
\hline Survival (days) & $91(26-222)$ & $323(86-394)$ & $P=0.002$ \\
\hline
\end{tabular}

Values are presented as medians with range (minimum-maximum) in parentheses unless otherwise stated.

P-values denote differences between the two groups referred and not referred for PN, using Kruskal-Wallis test for non-parametric variables and Chi2 test for categorical variables.

\section{Symptom Control}

Table 6 presents medications that were prescribed during each admission for the control of BO symptoms. When the palliative care team was involved, it provided advice regarding symptom control.

Table 6. Symptom control (medications prescribed during admission) by referral for PN and palliative care. 


\begin{tabular}{|c|c|c|c|c|c|c|c|}
\hline $\begin{array}{l}\text { Medication prescribed } \\
(\mathrm{n}, \%)\end{array}$ & $\begin{array}{c}\text { Total number of } \\
\text { admissions } \\
n=117\end{array}$ & $\begin{array}{c}\text { Referred } \\
\text { for PN } \\
n=48 \\
\end{array}$ & $\begin{array}{l}\text { Not referred } \\
\text { for PN } \\
n=69\end{array}$ & P-value & $\begin{array}{c}\text { Referred to } \\
\text { palliative care } \\
n=48\end{array}$ & $\begin{array}{l}\text { Not referred to } \\
\text { palliative care } \\
\quad n=51\end{array}$ & P-value \\
\hline $\begin{array}{l}\text { High dose steroids } \\
\quad \text { (dexamethasone }>7 \mathrm{mg} \text { ) }\end{array}$ & $47(40.2)$ & $20(41.7)$ & $27(39.1)$ & 0.783 & $26(54.2)$ & $13(25.5)$ & 0.004 \\
\hline \multicolumn{8}{|l|}{ Anti-emetics } \\
\hline Parenteral anti-emetics - Yes & $108(92.3)$ & $45(93.8)$ & $63(91.3)$ & 0.625 & $47(97.2)$ & $46(90.2)$ & 0.108 \\
\hline Cyclizine - Yes & $70(59.8)$ & $26(54.2)$ & $44(63.8)$ & 0.297 & $36(75.0)$ & $26(51.0)$ & 0.014 \\
\hline Levomepromazine - Yes & $49(41.9)$ & $21(43.8)$ & $28(40.6)$ & 0.732 & $28(58.3)$ & $13(25.5)$ & 0.001 \\
\hline Metoclopramide - Yes & $36(30.8)$ & $14(29.2)$ & $22(31.9)$ & 0.754 & $16(33.3)$ & $14(27.5)$ & 0.524 \\
\hline Number of anti-emetics & & & & 0.081 & & & $<0.0001$ \\
\hline 0 & $9(7.7)$ & $3(6.3)$ & $6(8.7)$ & & $1(2.1)$ & $5(9.8)$ & \\
\hline 1 & $38(32.5)$ & $13(27.1)$ & $25(36.2)$ & & $14(29.2)$ & $19(37.3)$ & \\
\hline 2 & $36(30.8)$ & $16(33.3)$ & $20(29.0)$ & & $10(20.8)$ & $22(43.1)$ & \\
\hline 3 & $28(23.9)$ & $16(33.3)$ & $12(17.4)$ & & $18(37.5)$ & $5(9.8)$ & \\
\hline 4 & $6(5.1)$ & $0(0.0)$ & $6(8.7)$ & & $5(10.4)$ & $0(0.0)$ & \\
\hline Morphine sulfate - Yes & $54(46.2)$ & $22(45.8)$ & $32(46.4)$ & 0.954 & $31(64.6)$ & $19(37.3)$ & 0.007 \\
\hline Oxycodone - Yes & $29(24.8)$ & $13(27.1)$ & $16(23.2)$ & 0.631 & $19(39.6)$ & $5(9.8)$ & 0.001 \\
\hline Fentanyl patch - Yes & $19(16.2)$ & $13(27.1)$ & $6(8.7)$ & 0.008 & $11(22.9)$ & $5(9.8)$ & 0.076 \\
\hline Diamorphine - Yes & $2(1.7)$ & $2(4.2)$ & $0(0.0)$ & 0.087 & $2(4.2)$ & $0(0.0)$ & 0.141 \\
\hline Alfentanil - Yes & $2(1.7)$ & $1(2.1)$ & $1(1.5)$ & 0.795 & $2(4.2)$ & $0(0.0)$ & 0.141 \\
\hline Number of opioids & & & & 0.067 & $5(10.4)$ & $26(51.0)$ & $<0.0001$ \\
\hline 0 & $39(33.3)$ & $15(31.2)$ & $24(34.8)$ & & $24(50.0)$ & $21(41.2)$ & \\
\hline 1 & $53(45.3)$ & $18(37.5)$ & $35(50.7)$ & & $16(33.3)$ & $4(7.8)$ & \\
\hline 2 & $22(18.8)$ & $12(25.0)$ & $10(14.5)$ & & $3(6.3)$ & & \\
\hline 3 & $3(2.6)$ & $3(6.3)$ & $0(0.0)$ & & & & \\
\hline Oral Opioid - Yes & $20(17.1)$ & $8(16.7)$ & $12(17.4)$ & 0.918 & $2(4.2)$ & $15(29.4)$ & 0.001 \\
\hline Parenteral paracetamol - Yes & $22(18.8)$ & $11(22.9)$ & $11(15.9)$ & 0.342 & $3(6.3)$ & $15(29.4)$ & 0.003 \\
\hline Oral paracetamol - Yes & $24(20.5)$ & $9(18.8)$ & $15(21.7)$ & 0.694 & $3(6.3)$ & $17(33.3)$ & 0.001 \\
\hline \multicolumn{8}{|l|}{ Laxatives } \\
\hline Laxatives - Yes & $79(67.5)$ & $30(62.5)$ & $49(71.0)$ & 0.333 & $32(66.7)$ & $36(70.6)$ & 0.674 \\
\hline Phosphate enema - Yes & $26(22.2)$ & $10(20.8)$ & $16(23.2)$ & 0.763 & $8(16.7)$ & $13(25.5)$ & 0.283 \\
\hline Lactulose - Yes & $21(18.0)$ & $7(14.6)$ & $14(20.3)$ & 0.429 & $7(14.6)$ & $11(21.6)$ & 0.368 \\
\hline Senna - Yes & $28(23.9)$ & $9(18.8)$ & $19(27.5)$ & 0.273 & $12(25.0)$ & $11(21.6)$ & 0.686 \\
\hline Microlette enema - Yes & $2(1.7)$ & $1(2.1)$ & $1(1.5)$ & 0.795 & $2(4.2)$ & $0(0.0)$ & 0.141 \\
\hline Arachis oil enema - Yes & $1(0.9)$ & $1(2.1)$ & $0(0.0)$ & 0.229 & $1(2.1)$ & $0(0.0)$ & 0.300 \\
\hline \multicolumn{8}{|l|}{ Anti-secretories } \\
\hline Parenteral anti-secretories - Yes & $28(23.9)$ & $14(29.2)$ & $14(20.3)$ & 0.268 & $20(41.7)$ & $4(7.8)$ & $<0.0001$ \\
\hline Hyoscine butylbromide - Yes & $26(22.2)$ & $12(25.0)$ & $14(20.3)$ & 0.547 & $18(37.5)$ & $4(7.8)$ & $<0.0001$ \\
\hline Octreotide - Yes & $6(5.1)$ & $6(12.5)$ & $0(0.0)$ & 0.003 & $6(12.5)$ & $0(0.0)$ & 0.005 \\
\hline Combination - Yes & $4(3.4)$ & $4(8.3)$ & $0(0.0)$ & 0.015 & $4(8.3)$ & $0(0.0)$ & 0.035 \\
\hline
\end{tabular}

Values are presented as frequencies with proportions in parentheses unless otherwise stated.

P-values denote differences between the two groups referred and not referred for PN, using Kruskal-Wallis test for non-parametric variables and Chi2 test for categorical variables.

High dose dexamethasone was prescribed in $40.2 \%$ patients with $8 \mathrm{mg}$ median dose (range 8-16mg). $66.7 \%$ patients were prescribed one or more parenteral opioids. The most common opioids prescribed were morphine sulfate (46.2\%) and oxycodone (24.8\%). 92.3\% patients were prescribed parenteral antiemetics. Of these $64.8 \%$ used combination therapy. The most frequently prescribed anti-emetics were cyclizine (59.8\%) and ondansetron (53.9\%). Laxatives were used in $67.5 \%$ patients, most commonly movicol (30.8\%) and sodium docusate (27.4\%). Injectable anti-secretories were used in 28 (23.9\%) patients. The types of parenteral anti-secretories prescribed were hyoscine butylbromide, octreotide, or a combination of both.

There were no significant differences in prescribing between PN group, other than in use of ondansetron $(p=0.02)$, and fentanyl patches $(p=0.008)$, which were associated with referral, and movicol, which was associated with non-referral $(p=0.019)$.

Referral to palliative care was associated with significant differences however. In total $48.5 \%$ of patients were referred to palliative care. Use of high dose steroids $(p=0.004)$, parenteral cyclizine $(p=0.014)$ and levomepromazine $(p=0.001)$, parenteral opioids $(p<0.0001)$, sodium docusate $(p=0.014)$ and parenteral anti-secretories $(p<0.0001)$ were all associated with referral to palliative care. In addition, referral to palliative care was associated with greater number of anti-emetics and parenteral opioids $(p<0.0001)$. Similarly, whether patients were conservatively managed or not was associated with higher use of levomepromazine (conservatively managed vs. not: $46 \%$ vs. $13 \%, P=0.016)$, oxycodone ( $28 \%$ vs. 
$0 \%, P=0.017)$; sodium docusate ( $31 \%$ vs. $0 \%, P=0.011)$; and senna $(27 \%$ vs. $0 \%, P=0.02)$

(Supplementary Table 2).

\section{Length of Stay and Readmissions}

Median length of stay was 13 days, with greater length of stay in those referred for PN than those who were not ( 28 vs. 9 day, $P=0.0001$ ). The median number of readmissions was 1 (range: $1-12$ ) andthere was no pattern as to whether readmissions were related to BO-, oncology-, or nutrition- reasons.

Median number of ITU admissions was 0 . Due to lack of data, we could not determine the place of death as there was missing data in approximately a third of the cohort. There were no re-admissions due to PN-related complications when using strict criteria.

\section{Survival}

Median (range) survival for this cohort of patients was: 20 (5.9-65.1) weeks (4.7 (1.4-15.2) months) from admission to death or censor date (Figure 1). In univariate analyses, more than one inpatient admission was associated with a statistically significant improved survival ( $\mathrm{HR}, 95 \% \mathrm{Cl}$ : $0.46(0.26-0.82)$, $\mathrm{P}=0.008$ ), whilst chemotherapy treatment (pre or during admission) was associated with a reduced survival $(2.74,1.22-6.16, \mathrm{P}=0.015)$ (Table 7 , Figure 2$)$. In addition, there was a survival advantage in those in the HPN group vs. those who may have required PN group (323 vs. 91 day, respectively $P=0.0021$ ) (Figure 3). There was no statistically significant association for other key factors (Supplementary Figures 2-8).

Figure 1. Kaplan Meier Curve for overall survival for patients with Malignant Bowel Obstruction. 


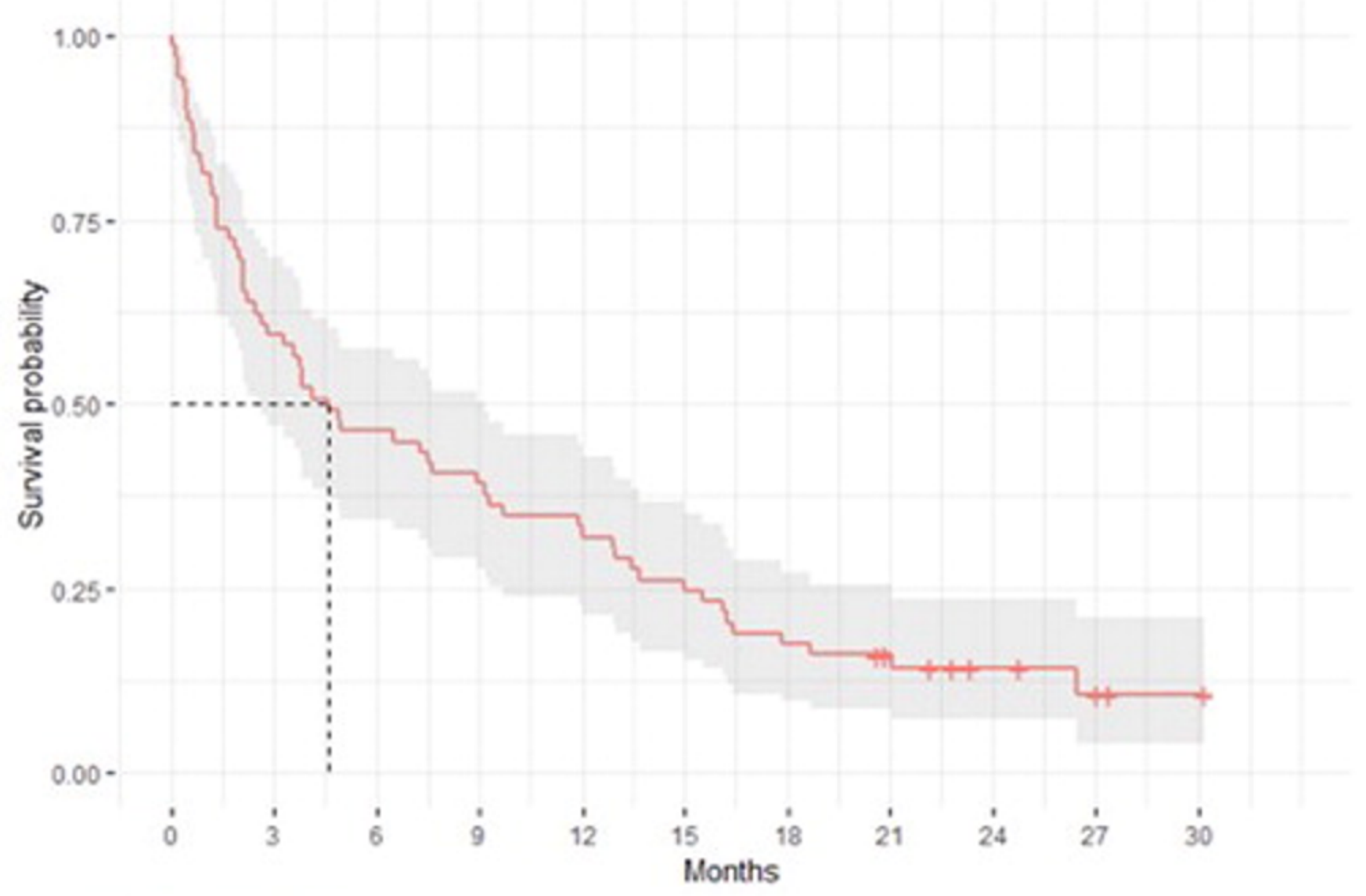

Number at risk

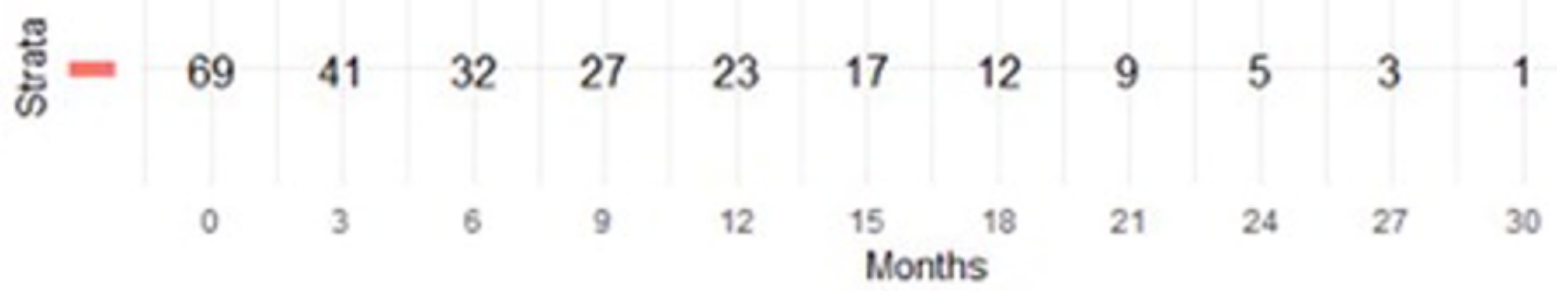

Figure 2. Kaplan Meier Curve by chemotherapy treatment for patients with Malignant Bowel Obstruction. 


\section{Chemotherapy + No $\mp$ Yes}
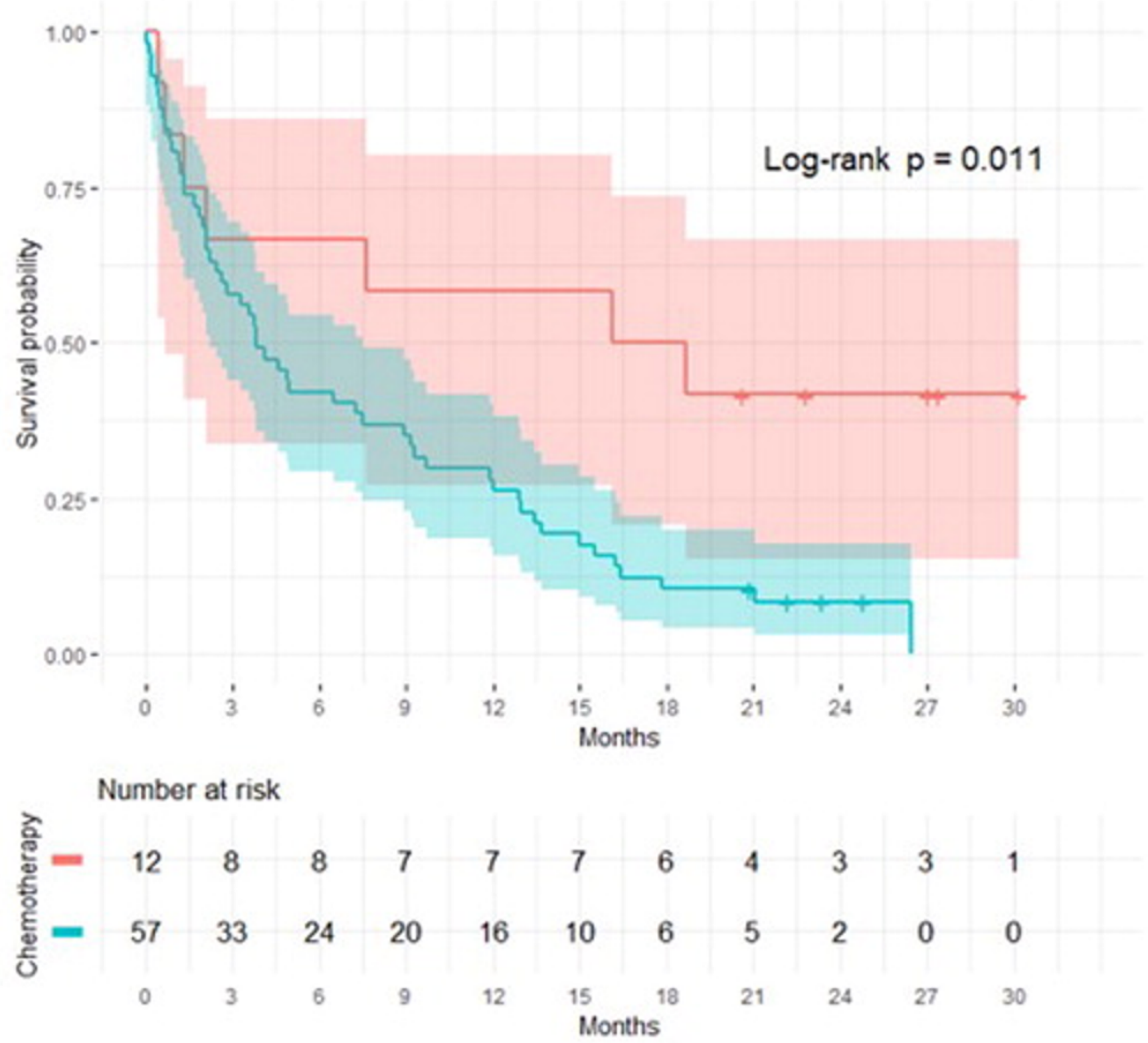

Figure 3. Kaplan Meier Curve by whether Malignant Bowel Obstruction patients 'may have' required PN vs. Patients who were discharged on home PN. 


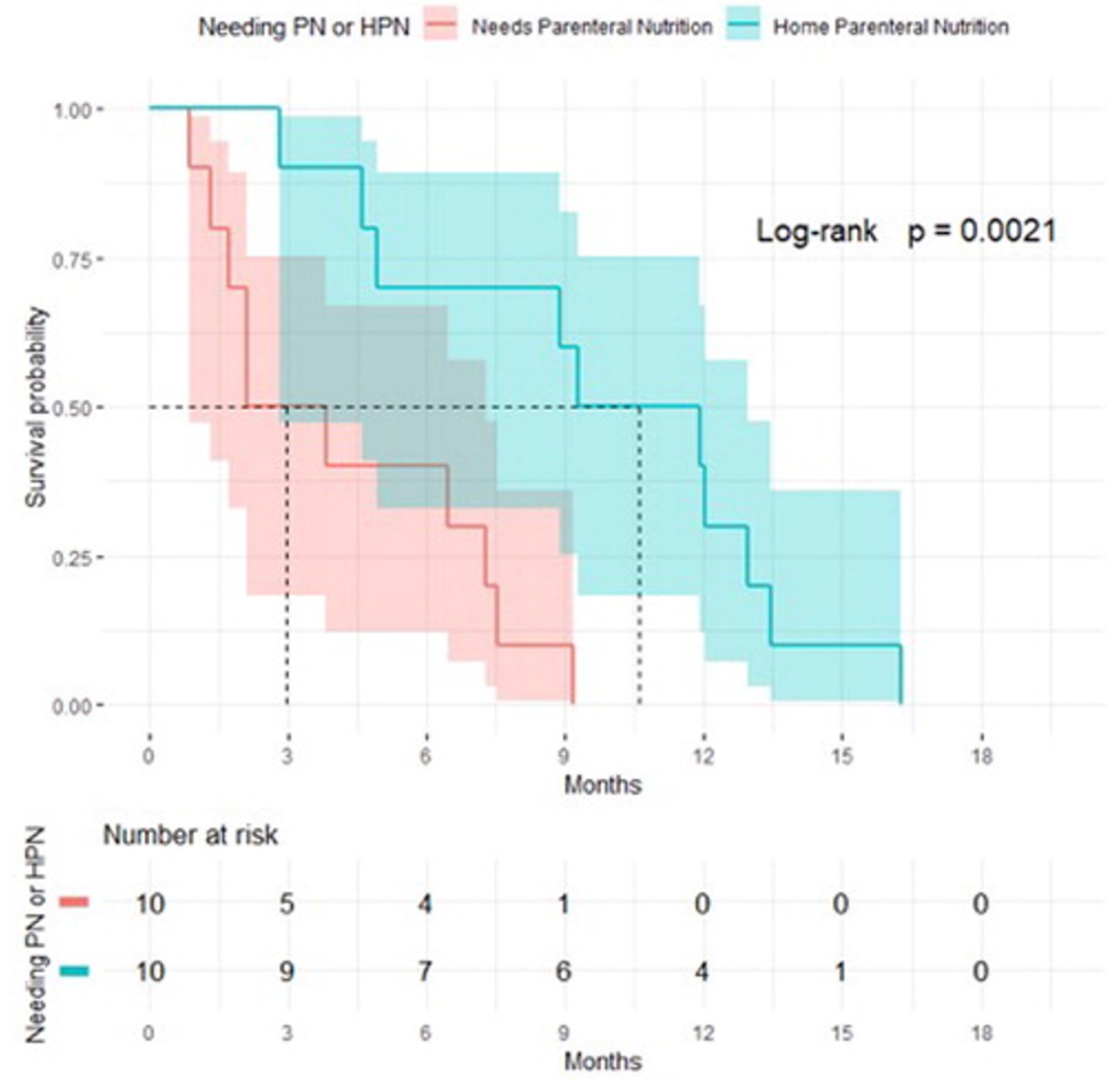

Table 7. Univariate analysis of key factors and their association with survival. 


\begin{tabular}{|c|c|c|c|}
\hline Variable & $\begin{array}{l}\text { Median survival (IQR) } \\
\text { (days) }\end{array}$ & Hazard ratio $(95 \% \mathrm{Cl})$ & P-Value \\
\hline \multicolumn{4}{|l|}{ Sex } \\
\hline Female & $109(36-410)$ & Ref. & \\
\hline Male & $232(140-641)$ & $0.58(0.30-1.12)$ & 0.107 \\
\hline \multicolumn{4}{|l|}{ Age } \\
\hline$<50$ years old & $271(140-568)$ & Ref. & \\
\hline$\geq 50$ years old & $109(36-394)$ & $1.61(0.82-3.18)$ & 0.170 \\
\hline \multicolumn{4}{|l|}{ Admission category } \\
\hline 1 admission & $67(27-296)$ & Ref. & \\
\hline$>1$ admission & 366 (229-499) & $0.46(0.26-0.82)$ & 0.008 \\
\hline \multicolumn{4}{|l|}{ Cancer diagnosis } \\
\hline Gynaecological & $100(27-366)$ & Ref. & \\
\hline Gastrointestinal & $222(109-491)$ & $0.70(0.40-1.24)$ & 0.221 \\
\hline Other & $75(36-641)$ & $0.72(0.32-1.63)$ & 0.428 \\
\hline \multicolumn{4}{|l|}{ Radiotherapy treatment } \\
\hline No & $140(52-473)$ & Ref. & \\
\hline Yes & $115(21-394)$ & $1.11(0.62-2.00)$ & 0.726 \\
\hline \multicolumn{4}{|l|}{ Chemotherapy treatment } \\
\hline No & $491(40-695)$ & Ref. & \\
\hline Yes & $117(41-393)$ & $2.74(1.22-6.16)$ & 0.015 \\
\hline \multicolumn{4}{|l|}{ Surgery for cancer } \\
\hline No & $109(41-456)$ & Ref. & \\
\hline Yes & $222(400-491)$ & $0.90(0.54-1.50)$ & 0.686 \\
\hline \multicolumn{4}{|l|}{ Location of bowel obstruction } \\
\hline Small & $117(57-410)$ & Ref. & \\
\hline Large & $232(41-499)$ & $0.90(0.46-1.74)$ & 0.752 \\
\hline Small and Large & $75(21-222)$ & $1.41(0.59-3.38)$ & 0.437 \\
\hline Unclear & $366(19-473)$ & $1.01(0.47-2.19)$ & 0.979 \\
\hline \multicolumn{4}{|l|}{ Type of bowel obstruction } \\
\hline Multi & $64(26-229)$ & Ref. & \\
\hline Single & $222(57-491)$ & $0.70(0.40-1.24)$ & 0.222 \\
\hline Unclear & $366(64-544)$ & $0.76(0.34-1.68)$ & 0.490 \\
\hline \multicolumn{4}{|l|}{ Inpatient $\mathrm{PN}$ received } \\
\hline No & $126(36-495)$ & Ref. & \\
\hline Yes & $140(75-393)$ & $1.19(0.68-2.08)$ & 0.543 \\
\hline \multicolumn{4}{|l|}{ HPN received } \\
\hline No & $115(36-491)$ & Ref. & \\
\hline Yes & $282(150-394)$ & $0.98(0.49-1.96)$ & 0.956 \\
\hline \multicolumn{4}{|l|}{ GPS } \\
\hline Score 0 & $117(27-456)$ & Ref. & \\
\hline Score 1 & $229(64-394)$ & $1.03(0.44-2.41)$ & 0.953 \\
\hline Score 2 & $80(26-495)$ & $0.96(0.40-2.29)$ & 0.927 \\
\hline \multicolumn{4}{|l|}{ NST score on admission } \\
\hline Low malnutrition risk $0-2$ & $296(67-641)$ & Ref. & \\
\hline Moderate malnutrition risk $3-6$ & $222(52-456)$ & $1.27(0.47-3.44)$ & 0.633 \\
\hline High malnutrition risk $\geq 7$ & $100(35-232)$ & $2.26(0.98-5.20)$ & 0.055 \\
\hline Weight loss on admission (kg) & & $0.99(0.97-1.02)$ & 0.548 \\
\hline \multicolumn{4}{|l|}{ Weight loss on admission (kg) } \\
\hline$<5 \%$ & $126(20-416)$ & Ref. & \\
\hline$\geq 5 \%$ & $140(64-394)$ & $0.87(0.47-1.64)$ & 0.673 \\
\hline BMI on admission $\left(\mathrm{kg} / \mathrm{m}^{2}\right)$ & & $1.02(0.96-1.08)$ & 0.527 \\
\hline BMI on admission $\left(\mathrm{kg} / \mathrm{m}^{2}\right)$ & & & \\
\hline$<18.5$ & $232(67-394)$ & Ref. & \\
\hline$\geq 18.5$ & $117(41-456)$ & $0.81(0.41-1.59)$ & 0.545 \\
\hline C-Reactive Protein (mg/L) & & $1.00(1.00-1.01)$ & 0.063 \\
\hline Albumin $(g / L)$ & & $0.99(0.96-1.03)$ & 0.731 \\
\hline White cell count $\left(x 10^{9} / \mathrm{L}\right)$ & & $1.00(0.99-1.01)$ & 0.748 \\
\hline Hemoglobin $(\mathrm{g} / \mathrm{L})$ & & $0.99(0.98-1.01)$ & 0.264 \\
\hline Sodium (mmol/L) & & $0.95(0.89-1.00)$ & 0.060 \\
\hline Potassium (mmol/L) & & $0.81(0.47-1.40)$ & 0.448 \\
\hline Urea (mmol/L) & & $1.03(0.98-1.09)$ & 0.240 \\
\hline Creatinine (mmol/L) & & $1.00(0.99-1.01)$ & 0.867 \\
\hline Magnesium $(\mathrm{mmol} / \mathrm{L})$ & & $0.35(0.03-4.54)$ & 0.423 \\
\hline Phosphate $(\mathrm{mmol} / \mathrm{L})$ & & $0.88(0.31-2.52)$ & 0.806 \\
\hline Adjusted calcium (mmol/L) & & $0.97(0.04-23.9)$ & 0.984 \\
\hline Ca 125 (kIU/L) & & $1.00(1.00-1.00)$ & 0.941 \\
\hline
\end{tabular}

Values are presented as median survival (interquartile range in parentheses) and unadjusted hazard ratios $(95 \% \mathrm{Cl}$ in parentheses) for each key factor.

Discussion

Summary of Key Findings 
This is the first study to examine care pathways of MBO patients by PN referral to the Nutrition Team. This MBO cohort was characterized by middle-aged women, who were more likely to have gynaecological or lower gastrointestinal primary malignancies with sub-diaphragmatical spread. Patients who were referred for PN were more likely to receive palliative chemotherapy and surgery for BO compared to those who were not referred. There were no differences in mGPS, NST score, or weight loss at admission for $\mathrm{MBO}$ in determining which patients were referred or not referred for PN. If patients were seen by a Dietitian or PN was discussed at MDT they were more likely to be referred for $\mathrm{PN}$. In $16 \mathrm{MBO}$ admissions (from 10 patients), the reasons for non-referral to the Nutrition team were unclear. We feel some of these patients 'may have' required PN due to: multiple admissions with BO, patients nil by mouth for more than 7 day and the ward dietitian querying the need for PN. Not all referred patients were started on PN based on assessment of indication/patient's wishes. There was a survival advantage in patients discharged on HPN vs. those who we speculate 'may have' required PN. There was a difference in symptom(s) control prescribing by whether a patient had been referred to palliative care, but not by referral to the nutrition team. Finally, increased length of stay was associated with those who were referred and received PN.

\section{Inpatient Parenteral Nutrition}

An important finding for patients who were referred for PN was that they were more likely to have PN discussed at the multidisciplinary meeting and/or have dietetic contact. This highlights the need for a standardized pathway for MBO patients in an MDT environment, which would incorporate nutrition into cancer management plans and facilitate open discussions around the risks vs. benefits of PN for the patient.

Additionally, in some cases it was unclear why patients were not referred for PN. There are strong (level 3) recommendations from ESPEN that state, in cancer patients, when oral and enteral routes have been exhausted, PN should be offered (22). While the general medical council provide guidance that artificial nutrition may be inappropriate toward the end of life, this can often extend to patients who are not amenable for further cancer treatment but have prognosis of $>3$ months. Several guidelines suggest that PN and HPN in the advanced cancer stage should be chosen on several criteria, albeit with nuances, involving the patient and wider MDT in the decision making $(22,23)$. A key finding of our study was a trend for some patients under the care of certain oncologists to be referred for PN. While the latter finding may be due to the retrospective nature of the study and missing information in some cases, it does reinforce the need for standardized pathways for the management of $\mathrm{MBO}$, to ensure every patient has the same access to treatment and support, when appropriate. For the 16 admissions (10 patients) that were not referred and it was speculated that they 'may have' required PN, we found that they tended to be under the care of certain oncologists $(1,2$, and 4$)$, were more likely to be female and have a gynaecological primary malignancy with metastases. These patients were likely to present with a moderate malnutrition risk, the majority were likely to be seen by a dietitian but did not have PN discussed at their MDT.

Unlike previous studies we could not identify traditional risk factors of survival such as albumin, mGPS, or KPS in our cohort (24-27). However, we did find that receiving chemotherapy as an inpatient was associated with poorer survival, which likely reflects disease severity. Higher malnutrition screening score on admission was also associated with poorer survival in our study. Malnutrition has been found to predict poor survival in advanced cancer patients (28), further highlighting the need that malnutrition should be assessed and treated earlier (29). It should be noted that a longer length of stay was observed for patients who received PN in our cohort suggesting that an earlier referral and a standardized pathway is needed to accelerate the safe set-up of HPN. Positively we did not observe any readmissions related to PN complications. We found no differences in routine admission biochemical markers, including the mGPS score by PN referral group, suggesting that most MBO patients present similarly with regards to biochemical markers, regardless of their need for PN. Most patients had a mGPS of 1 or 
two on admission suggesting poor prognosis which is evident in the relatively short survival of our MBO cohort.

\section{Symptom Control and Medical Management}

$\mathrm{MBO}$ was managed through a variety of pathways which likely reflects the clinical heterogeneity within the MBO cohort.

$50 \%$ patients were referred and seen by the surgical team, presumably where there was thought to be scope for surgical intervention or more urgent complications. Surgery, either open or laparoscopic, is usually reserved for patients with one level of obstruction, or small volume of disease, who are fit for such procedure. Stenting is recommended for patients in whom a radiologically diagnosed and endoscopically targetable lesion is identified (30). There is currently limited strong evidence on which surgical practice is based (14), and this patient-type are often not fit for either surgery or stenting (30). Thus unsurprisingly conservative management for $\mathrm{MBO}$ amongst the cohort was more common with over $80 \%$ of the cohort being managed conservatively (without open surgery) with ryles tube, high dose steroids, and/or symptom(s) control medications.

In this study, symptom control was managed by the primary (oncology or surgical) team with/without support from palliative care. While there were no differences in the proportion of patients referred to palliative care by PN referral group, only $48.5 \%$ of patients were referred to palliative care in total. This may be concerning, given that the literature suggests that, just as nutritional care should be provided earlier in the cancer course, so too should palliative care. Studies have shown that earlier palliative care input is associated with improved quality of life, through symptom control, holistic needs assessment, identification of deteriorating condition and timely planning of care $(31,32)$.

No standardized UK guidelines for the pharmacological management of MBO exist, which has also been observed in other countries (33). However, various local guidelines are available, which focus on the importance of parenteral administration routes for antiemetics and opioid analgesics, and the use of parenteral anti-secretories for the management of high volume vomits (34). A Cochrane review examining the use of high dose steroids for MBO management suggested a trend toward resolution of MBO when using high dose steroids (35). The literature guides palliative care practice generally and may explain why referral to palliative care was associated with the parenteral use of opioids, steroids and anti-secretories. The use of specific anti-emetics and laxatives depend on whether the obstruction is partial or complete and may explain why there were fewer associations with individual medications for these groups.

This study showed a lack of consistency in pharmacological management of the symptoms of bowel obstruction. Standardized, evidence-based guidelines would help unify care for all and optimize symptom management and quality of life.

\section{Strengths and Limitations}

This is the first study to examine medical and nutritional care pathways in patients with MBO. We have comprehensively looked at a range of factors to allow us to characterize this specific patient cohort. In addition, this study has 1 year follow-up data. We fill an important research gap by examining characteristics and survival in patients who are not referred for PN support.

The retrospective nature of this cohort did not allow $100 \%$ of data collection due to missing data. In Phase II we plan to overcome this limitation by collecting data prospectively. The small sample size of this study led to a reduced power to detect significant associations. We are currently in Phases II/III of our research strategy to examine this data nationally, which will increase the sample size and provide stronger study findings. We found a prevalence of $\mathrm{MBO}$ much lower than that previously reported and 
this is likely to be an underestimate, as coding for $\mathrm{MBO}$ is the function of the physician who is allocating the codes to a patients' inpatient discharge summary. Furthermore, patients may present with MBO earlier in the advanced cancer stage which may be incorrectly misclassified, eg., as 'constipation'.

\section{Conclusion}

All patients with $\mathrm{MBO}$ presented with high risk of malnutrition on admission and less than half were referred to the Nutrition team. Interestingly, patients who received a dietetic review and/or when PN was discussed at MDT, were more likely to be referred for PN. It was unclear in some cases why patients were not referred for PN. There was a trend for PN referral/non-referral by some oncologists.

Weight at follow-up were similar by PN referral groups, suggesting that PN attenuated the weight loss that potentially would have occurred had they not received PN. We could not identify any pattern in admission biochemical markers by PN referral group suggesting we may need to use other factors to identify patients who are not referred but require PN. Symptom control varied within this MBO cohort highlighting the need for standardized protocol of MBO management and referral to palliative care. Lastly, receiving PN did increase length of stay suggesting that earlier discussion and referral to the nutrition team should be sought. Survival was significantly longer in patients discharged on HPN vs. those who 'may have' required PN.

Timing of referral and integration of nutritional care at MDT is crucial to ensure that the variation in management of $\mathrm{MBO}$ is overcome. Further research in larger settings and quality of life factors is required and clear guidelines are required for $\mathrm{MBO}$ management avoid variation and ensure equality in access to PN when appropriate.

\section{Acknowledgments}

We would like to thank all members of the Intestinal Failure service at University College London Hospital.

\section{Author Contributions}

SDC conceptualized and supervised the study. PSP, KCF, KC, NK, contributed to analysis and wrote the manuscript. PSP, KC, NK, FOH collected data. All authors contributed to interpretation of data, drafting of the manuscript, and final approval of the manuscript for publication.

\section{Disclosure Statement}

P Patel's research post was funded by Calea, Fresenius Kabi, UK. All other authors have declared no conflict of interest.

\section{References}

Arends J, Baracos V, Bertz H, Bozzetti F, Calder PC, Deutz NEP, Erickson N, Laviano A, Lisanti MP, Lobo $D N$, et al. ESPEN expert group recommendations for action against cancer-related malnutrition. Clin Nutr. 2017;36(5):1187-96. doi:10.1016/j.clnu.2017.06.017

Fearon K, Arends J, Baracos V. Understanding the mechanisms and treatment options in cancer cachexia. Nat Rev Clin Oncol. 2013;10(2):90-9. doi:10.1038/nrclinonc.2012.209

Tsoli M, Robertson G. Cancer cachexia: malignant inflammation, tumorkines, and metabolic mayhem. Trends Endocrinol Metab. 2013;24(4):174-83. doi:10.1016/j.tem.2012.10.006

Bing C. Lipid mobilization in cachexia: mechanisms and mediators. Curr Opin Support Palliat Care. 2011;5(4):356-60. doi:10.1097/SPC.0b013e32834bde0e 
Slaviero KA, Read JA, Clarke SJ, Rivory LP. Baseline nutritional assessment in advanced cancer patients receiving palliative chemotherapy. Nutr Cancer. 2003;46(2):148-57. doi:10.1207/S15327914NC4602_07 Takudar R, Bruera E. Cachexia. In: Abeloff MD, Armitage J (eds): Clinical oncology. 3rd ed. Philadelphia (PA): Churchill Livingstone; 2004.

Martin L, Senesse P, Gioulbasanis I, Antoun S, Bozzetti F, Deans C, Strasser F, Thoresen L, Jagoe RT, Chasen $\mathrm{M}$, et al. Diagnostic criteria for the classification of cancer-associated weight loss. JCO. 2015;33(1):90-9. doi:10.1200/JCO.2014.56.1894

Caccialanza R, Cereda E, Pinto C, Cotogni P, Farina G, Gavazzi C, Gandini C, Nardi M, Zagonel V, Pedrazzoli $P$, et al. Awareness and consideration of malnutrition among oncologists: Insights from an exploratory survey. Nutrition. 2016;32(9):1028-32.: doi:10.1016/j.nut.2016.02.005

Muscaritoli M, Molfino A, Laviano A, Rasio D, Rossi Fanelli F. Parenteral nutrition in advanced cancer patients. Crit Rev Oncol Hematol. 2012;84(1):26-36. doi:10.1016/j.critrevonc.2012.01.005

Rauh S, Antonuzzo A, Bossi P, Eckert R, Fallon M, Fröbe A, Gonella S, Giusti R, Lakatos G, Santini D, et al. Nutrition in patients with cancer: a new area for medical oncologists? A practising oncologist's interdisciplinary position paper. ESMO Open. 2018;3(4):e000345: doi:10.1136/esmoopen-2018-000345 Ripamonti C, Twycross R, Baines M, Bozzetti F, Capri S, De Conno F, Gemlo B, Hunt TM, Krebs HB, Mercadante $S$, et al. Clinical-practice recommendations for the management of bowel obstruction in patients with end-stage cancer. Support Care Cancer. 2001;9(4):223-33. doi:10.1007/s005200000198 Anthony T, Baron T, Mercadante S, Green S, Chi D, Cunningham J, Herbst A, Smart E, Krouse RS. Report of the clinical protocol committee: Development of randomized trials for malignant bowel obstruction. J Pain Symptom Manage. 2007;34(1 Suppl):S49-S59. doi:10.1016/j.jpainsymman.2007.04.011 Tuca A, Guell E, Martinez-Losada E, Codorniu N. Malignant bowel obstruction in advanced cancer patients: epidemiology, management, and factors influencing spontaneous resolution. Cancer Manag Res. 2012;4:159-69. doi:10.2147/CMAR.S29297

Cousins SE, Tempest E, Feuer DJ. Surgery for the resolution of symptoms in malignant bowel obstruction in advanced gynaecological and gastrointestinal cancer. Cochrane Database Syst Rev. 2016;1:CD002764. Ferguson HJM, Ferguson Cl, Speakman J, Ismail T. Management of intestinal obstruction in advanced malignancy. Ann Med Surg (Lond). 2015;4(3):264-70. doi:10.1016/j.amsu.2015.07.018 Sowerbutts AM, Lal S, Sremanakova J, Clamp A, Todd C, Jayson GC, Teubner A, Raftery A-M, Sutton EJ, Hardy L, et al. Home parenteral nutrition for people with inoperable malignant bowel obstruction. Cochrane Database Syst Rev. 2018;8:CD012812: doi:10.1002/14651858.CD012812.pub2

Cotogni P, De Carli L, Passera R, Amerio ML, Agnello E, Fadda M, Ossola M, Monge T, De Francesco A, Bozzetti $F$, et al. Longitudinal study of quality of life in advanced cancer patients on home parenteral nutrition. Cancer Med. 2017;6(7):1799-806. doi:10.1002/cam4.1111

Baxter JP, Gillanders L, Angstmann K, Staun M, O'Hanlon C, Smith T, Joly F, Thul P, Jonkers C, Wanten G, et al. Prevalence of HPN in different countries. e-SPEN J. 2012;7(5):e211-e214.:

doi:10.1016/j.clnme.2012.08.001

Smith T, Naghibi M. 2016. BANS Report 2016: Artificial nutrition support in the UK 2005-2015, Adult home parenteral nutrition \& home intravenous fluids. 1st ed. [eBook] BAPEN (British Association of Parenteral and Enteral Nutrition) p. 10. http://www.bapen.org.uk.

McMillan DC, Crozier JEM, Canna K, Angerson WJ, McArdle CS. Evaluation of an inflammation-based prognostic score (GPS) in patients undergoing resection for colon and rectal cancer. Int J Colorectal Dis. 2007;22(8):881-6. doi:10.1007/s00384-006-0259-6

Karnofsky DA, Abelmann WH, Craver LF, Burchenal JH. The use of the nitrogen mustards in the palliative treatment of carcinoma. Cancer. 1948;1(4):634-56. doi:10.1002/1097-0142(194811)1:4<634::AIDCNCR2820010410>3.0.CO;2-L

Arends J, Bachmann P, Baracos V, Barthelemy N, Bertz H, Bozzetti F, Fearon K, Hütterer E, Isenring E, Kaasa $S$, et al. ESPEN guidelines on nutrition in cancer patients. Clin Nutr. 2017;36(1):11-48.

doi:10.1016/j.clnu.2016.07.015

August DA, Huhmann MB, American Society for Parenteral and Enteral Nutrition (A.S.P.E.N.) Board of Directors. A.S.P.E.N. clinical guidelines: Nutrition support therapy during adult anticancer treatment and in hematopoietic cell transplantation. JPEN J Parenter Enteral Nutr. 2009;33(5):472-500.

doi:10.1177/0148607109341804 
Pasanisi F, Orban A, Scalfi L, Alfonsi L, Santarpia L, Zurlo E, Celona A, Potenza A, Contaldo F. Predictors of survival in terminal-cancer patients with irreversible bowel obstruction receiving home parenteral nutrition. Nutrition. 2001;17(7-8):581-4. doi:10.1016/s0899-9007(01)00579-2

Santarpia L, Alfonsi L, Pasanisi F, De Caprio C, Scalfi L, Contaldo F. Predictive factors of survival in patients with peritoneal carcinomatosis on home parenteral nutrition. Nutrition. 2006;22(4):355-60. doi:10.1016/j.nut.2005.06.011

Laird BJ, Kaasa S, McMillan DC, Fallon MT, Hjermstad MJ, Fayers P, Klepstad P. Prognostic factors in patients with advanced cancer: A comparison of clinicopathological factors and the development of an inflammation-based prognostic system. Clin Cancer Res. 2013;19(19):5456-64. doi:10.1158/10780432.CCR-13-1066

Bozzetti F, Cotogni P, Lo Vullo S, Pironi L, Giardiello D, Mariani L. Development and validation of a nomogram to predict survival in incurable cachectic cancer patients on home parenteral nutrition. Ann Oncol. 2015;26(11):2335-40. doi:10.1093/annonc/mdv365

Yim GW, Eoh KJ, Kim SW, Nam EJ, Kim YT. Malnutrition identified by the nutritional risk index and poor prognosis in advanced epithelial ovarian carcinoma. Nutr Cancer. 2016;68(5):772-9.

doi:10.1080/01635581.2016.1159702

Aapro M, Arends J, Bozzetti F, Fearon K, Grunberg SM, Herrstedt J, Hopkinson J, Jacquelin-Ravel N, Jatoi $A$, Kaasa $S$, et al. Early recognition of malnutrition and cachexia in the cancer patient: a position paper of a European School of Oncology Task Force. Ann Oncol. 2014;25(8):1492-9.

doi:10.1093/annonc/mdu085

Laval G, Marcelin-Benazech B, Guirimand F, Chauvenet L, Copel L, Durand A, Francois E, Gabolde M, Mariani $P$, Rebischung $C$, et al. Recommendations for bowel obstruction with Peritoneal Carcinomatosis. J Pain Symptom Manage. 2014;48(1):75-91. doi:10.1016/j.jpainsymman.2013.08.022

Temel JS, Greer JA, El-Jawahri A, Pirl WF, Park ER, Jackson VA, Back AL, Kamdar M, Jacobsen J, Chittenden $\mathrm{EH}$, et al. Effects of early integrated palliative care in patients with lung and $\mathrm{Gl}$ cancer: $\mathrm{A}$ randomized clinical trial. J Clin Oncol. 2017;35(8):834-41.: doi:10.1200/JCO.2016.70.5046

Murray SA, Kendall M, Mitchell G, Moine S, Amblàs-Novellas J, Boyd K. Palliative care from diagnosis to death. BMJ. 2017;356:j878doi:10.1136/bmj.j878

Mittal DL, Mittal A, Brosnan EA, Landman WJ. Nonopioid pharmacological management of malignant bowel obstruction: A New Zealand-wide survey. J Palliat Med. 2014;17(11):1249-55.

doi:10.1089/jpm.2014.0176

Scottish Palliative Care Guidelines - Bowel Obstruction.

https://www.palliativecareguidelines.scot.nhs.uk/guidelines/symptom-control/bowel-obstruction.aspx. Feuer DJ, Broadley KE. Corticosteroids for the resolution of malignant bowel obstruction in advanced gynaecological and gastrointestinal cancer. Cochrane Database Syst Rev. 2000;2:CD001219. 